Anaesthesist $2011 \cdot 60: 797-798$

DOI 10.1007/s00101-011-1940-x

Online publiziert: 15. September 2011

(c) Springer-Verlag 2011

\section{J.C. Kubitz}

Klinik und Poliklinik für Anästhesiologie, Zentrum für Anästhesiologie und Intensivmedizin, Universitätsklinikum Hamburg-Eppendorf

\title{
Anästhesie bei Herzklappenerkrankungen
}

\section{Plädoyer für den perioperativen Anästhesiologen}

Erkrankungen, die zeitlich und örtlich gehäuft vorkommen, bezeichnet man allgemein als Epidemie. Im 21. Jh. wurde der Begriff der Epidemie, der ursprünglich Infektionskrankheiten vorbehalten war, auf Erkrankungen der Wohlstandsgesellschaft, wie den Diabetes oder die Adipositas, übertragen. Die Erkrankungen jedoch, die in Deutschland mit Abstand am häufigsten zu einer Aufnahme ins Krankenhaus führen, betreffen das kardiovaskuläre System. Laut den Diagnosedaten für 2009 des Statistischen Bundesamts (- Abb. 1; [1]) ist die Herzinsuffizienz nach der Geburt die häufigste Diagnose im Krankenhaus. Hinzu kommen unter den häufigsten Diagnosen weitere kardiale Erkrankungen wie die Angina pectoris, der Myokardinfarkt und das Vorhofflimmern, sodass nahezu 40\% der Diagnosen kardialen Ursprungs sind. In den Vereinigten Staaten von Amerika sind mehr als 5 Mio. Menschen von der Herzinsuffizienz betroffen; hier sprechen einige $\mathrm{Au}$ toren bereits von der „Herzinsuffizienzepidemie“ [2]. Doch trifft der Begriff der Epidemie auf diese Erkrankungen wirklich zu, wenn ein zeitliches und örtliches Ende angesichts der global weiter zunehmenden Prävalenz nicht in Sicht ist? Si- cher ist: Die kardiovaskulären Erkrankungen sind nicht nur von zentralem Interesse für die Gesundheitswissenschaften („public health“), sondern auch für den perioperativ tätigen Anästhesiologen.

In einer zunehmend alternden Bevölkerung haben in den letzten Dekaden die Prävalenz der Herzerkrankungen und, damit einhergehend, auch die Prävalenz der Herzklappenerkrankungen in der perioperativen Medizin stetig zugenommen. In einer vor wenigen Jahren erschienenen Publikation von van Klei et al. [3] wurden 2522 nichtkardiochirurgische Patienten evaluiert, bei denen in 4,2\% der Fäl-
Abb. 1 Diagnosedaten der Patienten in Krankenhäusern im Jahr 2009. (Angaben gemäß [1])

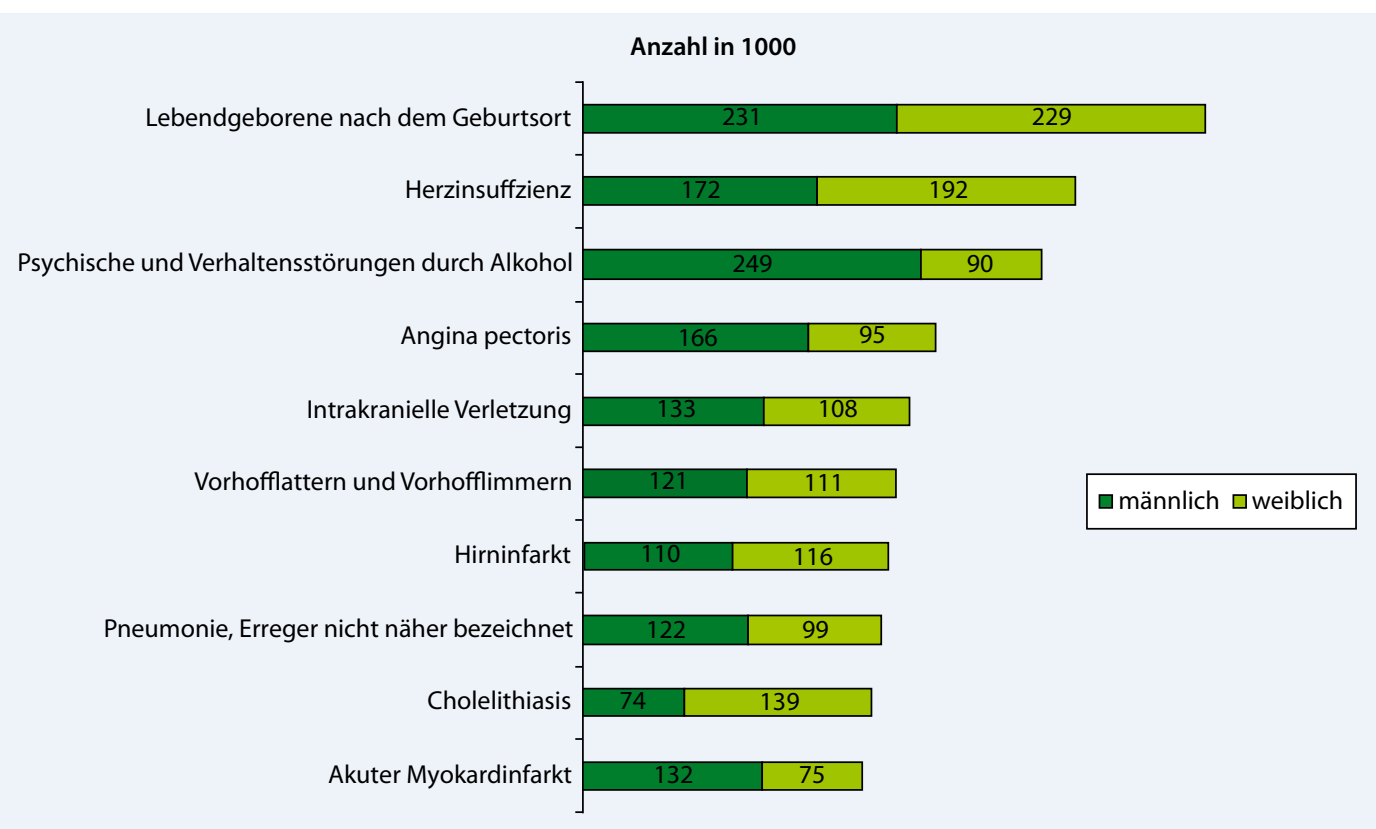


le bei der klinischen Untersuchung ein Herzgeräusch festgestellt worden war. Bei 70\% der Patienten mit Herzgeräusch lag diesem eine Herzklappenerkrankung zugrunde. Dies bedeutet, dass etwa bei jedem 30. erwachsenen Patient eine ihm nichtbekannte Herzklappenerkrankung vorliegt. Bei Patienten über 75 Jahren steigt die Prävalenz auf über 13\% [4].

In Zeiten, in denen die Sicherheit der Anästhesie v. a. angesichts der stets mehr werdenden Patienten mit Status III und IV in der Klassifikation der American Society of Anesthesiologists (ASA) Gegenstand einer interdisziplinären Diskussion ist [5], ist es von außerordentlicher Wichtigkeit, sich mit Erkrankungen, wie den Herzklappenerkrankungen, die entscheidend Einfluss auf das langfristige „outcome“ nehmen, aus dem Blickwinkel eines perioperativ tätigen Anästhesiologen auseinanderzusetzen. Insofern ist es sehr begrüßenswert, dass Mutlak et al. in dem Leitartikel dieser Ausgabe von Der Anaesthesist detailliert auf die anästhesiologische Versorgung von Patienten mit Herzklappenerkrankung von der Prämedikationsvisite bis hin zur postoperativen Betreuung eingehen. Ein grundlegendes Verständnis der pathophysiologischen Zusammenhänge bei Herzklappenerkrankungen ist hierbei unabdingbar. Mit der Anamnese und der klinischen Untersuchung, bei der die Einschätzung der körperlichen Belastbarkeit und die Beurteilung der kardialen Risikofaktoren auch für die Initiierung einer weiterführenden kardiologischen Diagnostik wegweisend ist [6], muss bereits die Erstellung eines individuellen perioperativen Behandlungsplans erfolgen. Dieser betrifft neben der Narkoseführung, unter Berücksichtigung eines möglichen Einsatzes von Regionalverfahren, und der Überwachung des Kreislaufs auch die perioperative Antikoagulation und die Endokarditisprophylaxe sowie die postoperative Nachbetreuung im „Intermediate-care“- oder Intensivbereich. Die postoperative Phase scheint sogar stärker mit Komplikationen behaftet zu sein als die intraoperative, wie eine retrospektive Analyse von $84 \mathrm{~Pa}$ tienten mit Mitralklappeninsuffizienz ergab. Dies ist wohl auf die engmaschigere intraoperative Überwachung und eine damit verbundene frühzeitige therapeuti- sche Intervention zurückzuführen. Ferner regen diese Ergebnisse natürlich die Diskussion an, ob auch Patienten mit einer Herzklappenerkrankung im mittleren Stadium von einer Korrektur des Herzvitiums vor einem elektiven, nichtkardiochirurgischen Eingriff profitieren [7]. Für eine generelle Empfehlung reicht hier die Datenlage nicht aus. Ein solches Vorgehen sollte im Einzelfall interdisziplinär diskutiert werden.

Eine frühzeitige differenzierte Volumen- und Katecholamintherapie trägt sicherlich zur Reduktion der intraoperativen Komplikationsrate bei, stellt aber auch die Weichen für ein günstiges postoperatives Outcome. Welches Monitoring-Verfahren jedoch zur Steuerung der Kreislauftherapie eingesetzt werden sollte, bleibt in vielen Fällen weiterhin offen. So konnte keines der gängigen Herzzeitvolumen(HZV)-Messverfahren, wie der Pulmonaliskatheter oder die Pulskonturanalyseverfahren $\left(\mathrm{PiCCO}^{\circledR}\right.$, Vigileo $^{\circledR}$, LID$\mathrm{CCO}^{\circledR}$ ) bei diesem Patientenkollektiv seine Überlegenheit beweisen. Hier besteht noch erheblicher Forschungsbedarf. Umso höher jedoch ist hier der Stellenwert der Echokardiographie als bettseitiges bildgebendes Verfahren, das zugleich Diagnosestellung und Evaluation der Therapiemaßnahmen ermöglicht. Nach einer kurzen (2-stündigen) theoretischen und praktischen Einweisung in die transthorakale Echokardiographie war es Anästhesiologen in Ausbildung möglich, eine klinisch signifikante Aortenstenose sicher zu diagnostizieren [8]. Angesichts der oben beschriebenen Zunahme herzkranker Patienten sollte die Etablierung mehr echokardiographischer Kompetenz im prä- sowie auch intraoperativen Bereich, ebenso aber auch auf den Intensivstationen $[9,10]$ zu den Zielen gehören, die im Rahmen der Entwicklung einer hochqualitativen perioperativen Anästhesiologie verfolgt werden. Die Deutsche Gesellschaft für Anästhesiologie und Intensivmedizin (DGAI) unterstützt seit mehr als einem Jahrzehnt diese Entwicklung durch interne Fortbildungsmaßnahmen zur Erlangung des Zertifikats „Transösophageale Echokardiographie in Anästhesiologie und Intensivmedizin“.

„Anästhesie bei Patienten mit Herzklappenerkrankung“ - dies betrifft uns al- le täglich. Um diesen Patienten ein größtmögliches Maß an Sicherheit zu bieten, muss die anästhesiologische Versorgung die gesamte perioperative Phase berücksichtigen. Der Beitrag von Mutlak et al. bietet einen praxisnahen Einstieg dazu, wie dies zu erreichen ist.
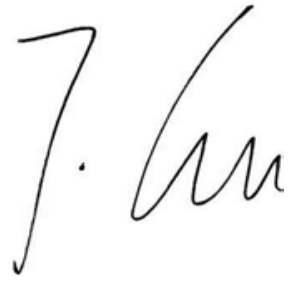

J.C. Kubitz

\section{Korrespondenzadresse \\ PD Dr. J.C. Kubitz}

Klinik und Poliklinik für Anästhesiologie, Zentrum für Anästhesiologie

und Intensivmedizin,

Universitätsklinikum Hamburg-Eppendorf

Martinistr. 52, 20246 Hamburg

jkubitz@uke.de

\section{Literatur}

1. Statistisches Bundesamt (2011) Gesundheit. Diagnosedaten der Patienten und Patientinnen. http://www.destatis.de/jetspeed/portal/cms/ Sites/destatis/Internet/DE/Content/Publikationen/Fachveroeffentlichungen/Gesundheit/Krankenhaeuser/DiagnosedatenKrankenhaus,templateld=renderPrint.psml

2. Roger VL (2010) The heart failure epidemic. Int J Environ Res Public Health 7:1807-1830

3. van Klei W, Kalkman CJ, Tolsma M et al (2006) Pre-operative detection of valvular heart disease by anaesthetists. Anaesthesia 61:127-132

4. Mittnacht AJ, Fanshawe M, Konstadt S (2008) Anesthetic considerations in the patient with valvular heart disease undergoing noncardiac surgery. Semin Cardiothorac Vasc Anesth 12:33-59

5. Gottschalk A, van Aken H, Zenz M et al (2011) Is anesthesia dangerous? Dtsch Arztebl Int 108: 469-474

6. Poldermans D, Bax JJ, Boersma E et al (2009) Guidelines for pre-operative cardiac risk assessment and perioperative cardiac management in non-cardiac surgery: the Task Force for Preoperative Cardiac Risk Assessment and Perioperative Cardiac Management in Non-cardiac Surgery of the European Society. Eur Heart J 30:2769-2812

7. Lai HC, Lai HC, Lee WL et al (2007) Mitral regurgitation complicates postoperative outcome of noncardiac surgery. Am Heart J 153:712-717

8. Cowie B, Kluger R (2011) Evaluation of systolic murmurs using transthoracic echocardiography by anaesthetic trainees. Anaesthesia 66:785-790

9. Vieillard-Baron A, Slama M, Cholley B et al (2008) Echocardiography in the intensive care unit: from evolution to revolution? Intensive Care Med 34:243-249

10. Expert Round Table on Ultrasound in ICU (2011) International expert statement on training standards for critical care ultrasonography. Intensive Care Med 37:1077-1083 